\title{
Correlations of Disk and Jet Emission Deviating from the Fundamental Plane
}

\author{
Da-Bin Lin ${ }^{1,2}$, Wei-Min $\mathrm{Gu}^{3}$, Hui-Jun $\mathrm{Mu}^{1,2}$, Zu-Jia Lu ${ }^{1,2}$, Ren-Yi Ma ${ }^{3}$, En-Wei Liang ${ }^{1,2}$
}

\begin{abstract}
The variability of accretion rate, which is believed to induce the aperiodic variability of X-ray emission from disk, may affect the energy injection into the jet. In this spirit, a correlation between disk emission and jet emission can be formed even if the mean luminosity of disk emission keeps constant. In this work, these correlations are found in the situation that the luminosity of disk emission is variable and kept with a constant mean value. The obtained correlations may be shallower than that of the fundamental plane of black hole activity. In addition, the slope of correlation may increase with increasing observed frequency of jet emission. For the luminosities spacing with three days, the slope of correlation decreases with increasing black hole mass. The deviation of our found correlations from that of the fundamental plane is related to the suppression of variability in the jet emission in comparison with that in the disk emission. This mechanism may work in some sources in which shallower correlations have been reported. Moreover, it implies that luminosities used to estimate the relation of fundamental plane should cover an appropriate timescale, in which the variability of jet emission is not significantly suppressed.
\end{abstract}

Subject headings: accretion, accretion disks - black hole physics - galaxies: active - galaxies: jets - X-rays: binaries

\section{Introduction}

The jet produced in an accreting black hole seems to be universal, since it is widely found in active galactic nuclei (AGNs) and Galactic black hole binaries (BHBs). The emission

\footnotetext{
${ }^{1}$ GXU-NAOC Center for Astrophysics and Space Sciences Department of Physics, Guangxi University, Nanning 530004, China; lindabin@gxu.edu.cn

${ }^{2}$ Guangxi Key Laboratory for Relativistic Astrophysics, the Department of Physics, Guangxi University, Nanning 530004, China;

${ }^{3}$ Department of Astronomy and Institute of Theoretical Physics and Astrophysics, Xiamen University, Xiamen, Fujian 361005, China
} 
from jets and accretion disks can be observed in the energy bands from radio to X-ray. For accretion systems with a jet, it is believed that the radio emission is mainly produced in the jet by the synchrotron process (Begelman et al. 1984). However, the X-ray emission is usually associated with the inner region of disk, where the temperature is highest and the corona may be formed (Liang \& Price 1977; Haardt \& Maraschi 1993). Since the jet is directly related to the accreting process, a correlation between radio and X-ray luminosities can be naturally expected. The existence of a tight, non-linear correlation between the radio $\left(L_{\mathrm{R}}\right)$ and X-ray luminaries $\left(L_{\mathrm{X}}\right)$, i.e., $L_{\mathrm{R}} \propto L_{\mathrm{X}}^{0.7 \pm 0.1}$, was found in BHBs during the low/hard state (Corbel et al. 2003; Gallo et al. 2003). This correlation was quickly extended to include AGNs, which is powered by accretion onto a super-massive black hole. The relation is the so-called "fundamental plane" of black hole activity, which can be described as $\log L_{\mathrm{R}}=\left(0.60_{-0.11}^{+0.11}\right) \log L_{\mathrm{X}}+\left(0.78_{-0.09}^{+0.11}\right) \log \left(M_{\mathrm{BH}} / M_{\odot}\right)+7.33$ (Merloni et al. 2003; Falcke et al. 2004; Merloni et al. 2006). Here, $L_{\mathrm{R}}$ is the nuclear radio luminosity at frequency $\nu=5 \mathrm{GHz}$, $L_{\mathrm{X}}$ is the nuclear X-ray luminosity at $2-10 \mathrm{keV}, M_{\mathrm{BH}}$ is the black hole mass, and $M_{\odot}$ is the solar mass. Such a plane confirms that the production of jets (traced by radio luminosity) is fundamentally associated with the accreting process (traced by X-ray luminosity).

The emission from accretion disks in BHBs and AGNs displays significant aperiodic variabilities on a broad range of timescales. The most promising explanation for these variabilities is the model of propagating fluctuations Lvubarskii 1997; King et al. 2004; Mayer \& Pringle 2006; Janiuk \& Czerny 2007; Lin et al. 2012). In this model, the variabilities of disk emission is induced by the fluctuations of accretion rate, which are caused at different radii and propagate into the inner region of the disk. The fluctuations of accretion rate in the inner region would modulate the energy released in the vicinity of black hole, where produces most of X-ray emission. Since the production of jets is fundamentally associated with the accreting process, the energy injected into the jet should be affected by the fluctuations of accretion rate. In this work, we present the variability of jet power and thus the jet emission in the model of propagating fluctuations. The effects of these variabilities on the relation of fundamental plane are our main focus.

This paper is organized as follows. The models for variability of disk emission and jet emission are presented in Section 2, The light curves produced based on the above models are shown in Section 3, Our main focus in the present work, i.e., the relations between disk emission and jet emission, are also discussed in this section. In Section 4, we summary conclusions and present a brief discussion. 


\section{Models}

\subsection{Variability of accretion disk emission}

The model of propagating fluctuations in mass accretion rate is proposed to explain the complex variability of emission from accreting disk (Lyubarskii 1997; Kotov et al. 2001; Arévalo \& Uttley 2006). In this picture, fluctuations stirred up far from the black hole modulate the mass accretion rate and thus the emission from the disk, which is mainly produced in the inner region of the disk. The fluctuations are produced on timescales related to the viscous timescale at the radius of its origin and are uncorrelated for different radial scales. In this scenario, the mixture of fluctuations with different timescales is a multiplicative process. Then, the emitted flux from accretion disk is naturally predicted to display a linear rms - flux relation, which is observed in a number of AGNs and BHBs (Uttley \& McHardy 2001; Uttley et al. 2005; Heil et al. 2012).

To generate light curves according to the model of propagating fluctuations, the energy release profile of accretion flow in the radial direction, which determines the emission behavior of disk, should be assumed. In this paper, $\epsilon(r)=r^{-3}\left(1-\sqrt{r_{\text {in }} / r}\right)$ is taken to follow the radial loss rate of gravitational energy in the accretion disk, where $r$ represents the radius, $r_{\text {in }}$ is the inner radius of the disk and is fixed at $3 r_{\mathrm{g}}$ in the following simulations, and $r_{\mathrm{g}}=2 G M_{\mathrm{BH}} / c^{2}$ is the Schwarzschild radius of black hole. In the standard thin accretion disc (Shakura \& Sunyaev 1973), the emitted flux per unit area, $F(r, t)$, is proportional to $\epsilon(r)$ and $\dot{M}(r, t)$, i.e., $F(r, t) \propto \dot{M}(r, t) \epsilon(r)$, where $\dot{M}(r, t)$ is the mass accretion rate at the radius $r$ and time $t$. In this situation, the luminosity of disk emission can be read as

$$
L_{\mathrm{X}}=\int_{r_{\mathrm{in}}}^{r_{\mathrm{out}}} F(r, t) 2 \pi r d r \propto \int_{r_{\mathrm{in}}}^{r_{\mathrm{out}}} \dot{M}(r, t) \epsilon(r) 2 \pi r d r .
$$

As pointed out by the Arévalo \& Uttley (2006), the emissivity profile of different photons may be different since the emitted spectrum might be radius-dependent. Then, we should modify the function of $\epsilon(r)$ to describe the emissivity profile of different photons. For this purpose, an adequate value of $\gamma$ can be introduced and the emissivity profile is modified as $\epsilon(r, \gamma)=r^{-\gamma}\left(1-\sqrt{r_{\text {in }} / r}\right)$ for different photons. To our knowledge, the X-ray emission may be produced in an accreting corona (Liang \& Price 1977), possibly sandwiching an optically thick, geometrically thin disk, or in the advection-dominated accretion flow (ADAF: Narayan \& Yi 1994; Yuan \& Narayan 2014). In the ADAF, which is the focused accretion flow in this work (see the discussion in Section 2.2), the emitted flux per unit area $F(r, t)$ is proportional to $M(r, t)^{2}$ rather than $\dot{M}(r, t)$. Then, Equation (1) can be turned into

$$
L_{\mathrm{X}} \propto \int_{r_{\mathrm{in}}}^{r_{\mathrm{out}}} \dot{M}(r, t)^{2} \epsilon(r, \gamma) r d r
$$


in the ADAF. This is the equation used to produce the light curves of X-ray emission from disk in this work. It is shown that the light curves produced with different $\gamma(\gamma>2.5)$ does not show significant difference except for the variability in the shortest timescales (see Figure 2 of Arévalo \& Uttley 2006), which does not affect the relations studied in this work (please see the discussion in Section 3.2). Then, we discuss the fluctuations of disk emission with $\gamma=3$.

\subsection{Variability of Jet Power}

The dominant paradigms for jet production are outlined in the works of Blandford \& Znajek (1977) (BZ model) and Blandford \& Payne (1982) (BP model). In both of the models, the large-scale ordered magnetic fields threading the accretion flow or spinning black hole are required. It is suggested that the large-scale magnetic fields may diffuse outward rapidly if the half-thickness of the accretion disk $H$ is significantly less than the radius $r$ (Lubow et al. 1994; Hevvaerts et al. 1996; Guan \& Gammie 2009). Then, the jet discussed in our paper should be produced in a geometrically thick disk (Maccarone et al. 2003; Sikora et al. 2007; Coriat et al. 2011; Russell et al. 2011), such as the ADAF or the slim disk (Abramowicz et al. 1988). Our work focuses on the jet produced in the ADAF, of which the accretion rate is relatively low. The jet power $\left(P_{\text {jet }}\right)$ in the $\mathrm{BP}$ and $\mathrm{BZ}$ models is associated with the strength of large-scale ordered poloidal magnetic field $B_{\mathrm{p}}$. In the BP model, the jet power can be described as (e.g., Livio et al. 1999; Cao 2002; Li \& Cao 2012)

$$
P_{\text {jet }} \sim \int_{r_{\text {in }}}^{r_{\mathrm{out}}} \Omega\left(r \frac{B_{\mathrm{p}}^{2}}{4 \pi}\right) w(r) 2 \pi r d r
$$

where $\Omega(r) \propto r^{-3 / 2}$ is the angular velocity of disk, $r B_{\mathrm{p}}^{2} /(4 \pi)$ is the torque applied on the disk, and $w(r) \propto r^{-\mu}$ is introduced to describe the contribution of different radius to the jet power. In the BP model, the energy of jet is mainly from the inner region of disk. Then, $\mu$ should be larger than or equal to 0, i.e.,

$$
\mu \gtrsim 0
$$

The strength of large-scale poloidal magnetic field remains uncertain, but is believed to be associated with the accreting process (e.g., Moderski \& Sikora 1996; Ghosh \& Abramowicz 1997; Livio et al. 1999; Meier 2001; Nemmen et al. 2007). In addition, the strength of smallscale magnetic field $\left(B_{\mathrm{p}, \mathrm{d}}\right)$ in the magnetohydrodynamic turbulence of the disk is usually used to estimate the strength of $B_{\mathrm{p}}$, i.e., $B_{\mathrm{p}} \sim B_{\mathrm{p}, \mathrm{d}}$ in the ADAF (Livio et al. 1999; Meier 2001; Wu \& Cao 2008). In the accretion flow, $B_{\mathrm{p}}$ may be radius-dependent. In order to 
model this behavior, we adopt (Livio et al. 1999; Meier 2001; Wu \& Cao 2008; Li \& Cao 2012)

$$
B_{\mathrm{p}}^{2} \propto \tau_{r \phi}\left(r / 3 r_{\mathrm{g}}\right)^{-s}
$$

where $\tau_{r \phi}=\left\langle\delta B_{r} \delta B_{\varphi} / 4 \pi-\rho \delta v_{r} \delta v_{\varphi}\right\rangle_{\varphi} \propto B_{\mathrm{p}, \mathrm{d}}^{2}$ is the stress in the magnetohydrodynamic accretion flow. If the parameter $s$ in Equation (5) is larger than $0, B_{\mathrm{p}}$ in the outer region will suppress the magnetorotational instability (Velikhov 1959; Chandrasekhar 1960; Balbus \& Hawley 1991, 1998), which is responsible for the angular momentum transport. Then, $s$ should be larger than or equal to 0 , i.e.,

$$
s \gtrsim 0
$$

Based on the above discussion, Equation (3) becomes

$$
P_{\text {jet }}(t)=\zeta \int_{r_{\text {in }}}^{r_{\text {out }}} \dot{M}(r, t) r^{-\mu-s} \epsilon(r, 3) r d r=\zeta \int_{r_{\text {in }}}^{r_{\text {out }}} \dot{M}(r, t) \epsilon(r, 3+\mu+s) r d r,
$$

where $H \sim r$ and $\Omega \tau_{r \varphi} H \propto \dot{M} \epsilon(r, 3)$ are taken and $\zeta$ is a constant. In the BZ model, the variability of jet power, which is induced by the fluctuations of magnetic field in the vicinity of black hole, does not present significant difference with Equation (17). Then, we use Equation (7) to describe the jet power. This equation reveals that the power of jet depends linearly on the accretion rate in the ADAF, which has been used in a number of works (e.g., Meier 2001; Nemmen et al. 2007; Martínez-Sansigre \& Rawlings 2011). Equation (7) is similar to Equation (11) but with $\gamma=3+\mu+s$ and $\mu+s \gtrsim 0$. It was discussed previously that the variabilities based on Equation (2) (or Equation (7)) with varying $\gamma(\gamma>2.5)$ do not show significant difference. Then, the variability of jet power based on Equation (7) would follow that of accretion rate or $L_{X}$ except for the amplitude of variability, which has been found in the observation of GX 339-4 (Casella et al. 2010). This fact implies that Equation (7) is applicable to describe the jet power. In our work, we take $\mu+s=0$ (please see the discussion in Section 3.2). In this situation, we take $\zeta=0.1$, which corresponds to the case that $10 \%$ of gravitational energy released in the accretion process enters the jet.

In the following part of this Section, we try to work out the variability of jet emission based on the jet power presented in Equation (7). The jet emission is modeled in the internal shock scenario, which has been widely adopted to explain the broadband spectral energy distribution of AGNs, BHBs, and the prompt emission of gamma-ray bursts (Piran 1999; Spada et al. 2001; Yuan et al. 2005; Wu et al. 2007; Nemmen et al. 2011; Sun et al. 2013). Accompanying with the accretion onto a black hole, a small fraction of the accreted material $\dot{M}_{\text {jet }}(t, z=0)=P_{\text {jet }}(t) /(\bar{\Gamma}-1) c^{2}$ is transferred into the vertical direction, where $z$ represents the distance measured along the jet with respect to the black hole. With the propagation of $\dot{M}_{\text {jet }}$ in this direction, the phenomena of jet are formed. Since $P_{\text {jet }}(t)$ varies 
with time, the value of $\dot{M}_{\text {jet }}(t, z)\left(=P_{\text {jet }}\left(t-z / v_{\text {jet }}\right) /(\bar{\Gamma}-1) c^{2}\right)$ may be different for different $z$ at the same time $t$, where $v_{\text {jet }}=c\left(1-\bar{\Gamma}^{-2}\right)^{1 / 2}$ is the velocity of jet and $c$ is the velocity of light. According to the internal shock model, the Lorentz factor of jet varies with time, and as a result, faster portions of the jet catch up with slower portions of the jet. The internal shocks are formed in the ensuing collision, which occurs in the region of $z \geqslant z_{0}$. In the shocks, a small fraction $\xi_{e}$ of the electrons are accelerated and form a power-law energy distribution with index $p$. The broad-band emission of jet is mainly produced by these electrons through synchrotron process (Markoff et al. 2001). In order to calculate the jet emission, the jet half-opening angle $\phi$, the average bulk Lorentz factor $\bar{\Gamma}$ of the jet, the angle $\psi$ of the jet with respect to the line of sight, and the energy density ratio $\epsilon_{e}\left(\epsilon_{B}\right)$ of accelerated electrons (amplified magnetic field) to the shock energy should be prescribed. In the present work, the calculation of jet emission follows the procedure presented in the Appendix of Yuan et al. (2005). The values of $\phi=0.1$ radians, $\bar{\Gamma}=1.2, \psi=30^{\circ}, \xi_{e}=1 \%$, $p=2.1, \epsilon_{e}=0.06$, and $\epsilon_{B}=0.02$ are adopted in our calculation. The average of $\dot{M}$ is taken as $1.39 \times 10^{16}\left(M_{\mathrm{BH}} / M_{\odot}\right) \mathrm{g} / \mathrm{s}=0.01 \dot{M}_{\text {Edd }}$, i.e., $\overline{\dot{M}}=0.01 \dot{M}_{\text {Edd }}$, where $\dot{M}_{\text {Edd }}$ is the Eddington accretion rate. In this paper, we focus on the variability of jet emission induced by the fluctuation of accretion rate, i.e., Equation (7). Then, the variability due to the collisions between shells in the internal shock model, which may increase the scattering of our studied correlations, is not considered in this work.

\section{Relations between disk emission and jet emission}

\subsection{Results}

Based on the models presented in Section 2, we produce the light curves of disk emission and jet emission as shown in Figure 1. The produced $L_{\mathrm{X}} / \overline{L_{\mathrm{X}}}$ and $P_{\text {jet }} / \overline{P_{\text {jet }}}$ are shown with green and black curves in this figure, and the emission of jet $L_{\text {jet }}(\nu) / \overline{L_{\text {jet }}(\nu)}$ at different frequency $\nu$ is described with different color curves: the blue curves are for $\nu=10^{10} \mathrm{~Hz}$, the gray curves for $\nu=10^{11} \mathrm{~Hz}$, the red curves for $\nu=10^{12} \mathrm{~Hz}$, and the cyan curves for $\nu=10^{13} \mathrm{~Hz}$. Here, $L_{\text {jet }}(\nu)$ represents the luminosity of jet emission at frequency $\nu$ and $\overline{L_{\{\ldots\}}}$ $\left(\overline{P_{\text {jet }}}\right)$ represents the mean value of $L_{\{\ldots\}}\left(P_{\text {jet }}\right)$. The light curves in the top (bottom) panel of this figure are produced in the accretion system with $M_{\mathrm{BH}}=10 M_{\odot}\left(M_{\mathrm{BH}}=10^{7} M_{\odot}\right)$ in a duration of $100\left(10^{8}\right)$ seconds, where the total duration of simulation $T_{\text {tot }}$ is around 1700 $\left(1.7 \times 10^{9}\right)$ seconds. It can be found that the light curves of disk emission are similar to those presented in the Figure 1 of Arévalo \& Uttley (2006). Moreover, the amplitude of variability in the jet emission at low frequency, such as $\nu=10^{10} \mathrm{~Hz}$, may be weak compared with that in the jet power (disk emission). However, the variability of jet emission at high frequency, 
such as $\nu=10^{13} \mathrm{~Hz}$, almost follows the variability of jet power. Because of those behaviors, some interesting relations between disk emission and jet emission may be formed. We should emphasize that the above behaviors are presented in the accretion system with a constant $\overline{\dot{M}}$ (or $\overline{L_{\mathrm{X}}}$ ) rather than an evolving $\overline{\dot{M}}$ (or $\overline{L_{\mathrm{X}}}$ ). In the following part, we discuss these relations.

In order to reduce the data points in the top panel of Figure 1, we divide the light curves with a bin size $(\Delta t)$ of one second and obtain the average luminosity in each bin as the new data. We analyse the relation of disk emission and jet emission based on these new data. The results are shown in Figure 2, which reveals the strong correlation between disk emission and jet emission. In this figure, the relation between disk emission and jet emission at different observed frequency $\nu$ is represented with different symbol. The symbol " $\triangle$ " is for the jet emission at frequency $\nu=10^{10} \mathrm{~Hz}$, " $\times$ " for $\nu=10^{11} \mathrm{~Hz}$, "०" for $\nu=10^{12} \mathrm{~Hz}$, and " $\star$ " for $\nu=10^{13} \mathrm{~Hz}$. In each panel of this figure, the solid line represents the logarithmic linear fitting result of the data, and the dashed line represents the relation of the fundamental plane with a given black hole mass, i.e., $\log \left(L_{\text {jet }} / \overline{L_{\text {jet }}}\right) \propto 0.60 \log \left(L_{\mathrm{X}} / \overline{L_{\mathrm{X}}}\right)$. According to this figure, it is easy to find that the relation of disk emission and jet emission at frequency $10^{10} \mathrm{~Hz}$ almost runs parallel with the abscissa axis, i.e., the axis of disk emission. This behavior is similar to that presented in Figure 5 of King et al. (2013), which suggests that the results in this work may provide an explanation for this kind of peculiar correlations. In addition, the slope of relation increases with increasing observed frequency of jet emission. The largest slope of these relations is reached by the jet emission at frequency $10^{13} \mathrm{~Hz}$ with a slope of 0.58 , close to the slope of the fundamental plane relation. This reveals that the relation of fundamental plane is only followed by the jet emission at frequency $10^{13} \mathrm{~Hz}$ for the situation studied here, i.e., $M_{\mathrm{BH}}=10 M_{\odot}, \Delta t=1 \mathrm{~s}$, and with a duration of $100 \mathrm{~s}$ observation.

Based on Figure 1, one may find that the amplitude of variability in the jet emission at the same frequency (such as $10^{10} \mathrm{~Hz}$ ) may be different for different black hole mass. Owing to this fact, we show the relations of disk emission and jet emission $\left(\nu=10^{10} \mathrm{~Hz}\right)$ for different black hole mass in Figure 3. In this figure, the symbols " $\star$ ", "०", " $\triangle$ ", and " $\times$ " represent the data (i.e., averaged luminosities in time span $\Delta t$ ) from the accretion system with $M_{\mathrm{BH}}=$ $10^{3} M_{\odot}\left(\Delta t=10^{2} \mathrm{~s}\right), 3.6 \times 10^{5} M_{\odot}\left(\Delta t=3.6 \times 10^{4} \mathrm{~s}\right), 10^{7} M_{\odot}\left(\Delta t=10^{6} \mathrm{~s}\right)$, and $10^{9} M_{\odot}(\Delta t=$ $\left.10^{8} \mathrm{~s}\right)$, respectively. In each panel of this figure, the solid line represents the logarithmic linear fitting result of the data, the dashed line represents the relation of fundamental plane with a given black hole mass, and the data are from $10 M_{\mathrm{BH}} / M_{\odot}$ seconds simulation $\left(T_{\text {tot }} \sim\right.$ $\left.170 M_{\mathrm{BH}} / M_{\odot} \mathrm{s}\right)$. According to this figure, the slope of relation between disk emission and jet emission increases with increasing black hole mass, where $\Delta t \propto M_{\mathrm{BH}}$ is adopted. The upper limit of the relation slope is 0.46 , close to the slope of the fundamental plane relation. In a practical context, the observations are usually performed in a short period (such as 1ks) and luminosities used to estimate the relation of fundamental plane are selected with an average 
spacing of several days. In order to model this situation, we average luminosities in $\Delta t=1 \mathrm{ks}$ as the new data and use the data with a spacing time of 3 days to estimate the relation of disk emission and jet emission. Figure 4 shows the relations for these luminosities, where data are from $1.5 \times 10^{7} \mathrm{~s}$ simulation $\left(T_{\text {tot }} \sim 1.2 \times 10^{8} \mathrm{~s}\right)$. In this figure, the symbols " $\star$ ", "०", " $\triangle$ ", and "×" represent the data from the accretion system with $M_{\mathrm{BH}}=10 M_{\odot}, 10^{3} M_{\odot}, 3.6 \times 10^{5} M_{\odot}$, and $10^{7} M_{\odot}$, respectively. Since the viscous timescale of the accretion disk around the black hole with $M_{\mathrm{BH}} \sim 10^{9} M_{\odot}$ is significantly larger than 1 hour, the accretion system with $M_{\mathrm{BH}} \sim 10^{9} M_{\odot}$ is not considered. Figure 4 shows that the slope of relations decreases with increasing black hole mass. The largest slope of these relations (0.63), found in the accretion system with $M_{\mathrm{BH}}=10 M_{\odot}$, is close to the slope of fundamental plane. That is to say the deviation of our relations from that of fundamental plane becomes significant for systems with more massive black hole in the case studied in Figure 4. For the comparison with the observations, a dotted line, i.e., $\log \left(L_{\text {jet }} / \overline{L_{\text {jet }}}\right) \propto 0.06 \log \left(L_{\mathrm{X}} / \overline{L_{\mathrm{X}}}\right)$, which describes the relation of disk emission and jet emission at $\nu=5 \mathrm{GHz}$ found in NGC 4395 (King et al. 2013), is added in the bottom left panel of this figure. In this panel, the red "** represents the data of $L_{\mathrm{X}}$ and $L_{\text {jet }}(\nu=5 \mathrm{GHz})$ based on the models in Section 2. The logarithmic linear fitting about these data presents a relation of $\log \left(L_{\text {jet }} / \overline{L_{\text {jet }}}\right) \propto 0.19 \log \left(L_{\mathrm{X}} / \overline{L_{\mathrm{X}}}\right)$, which is described with a red solid line in this panel. It is shown that our relation in the accretion system with $M_{\mathrm{BH}}=3.6 \times 10^{5} M_{\odot}$ is shallower than that of the fundamental plane, but steeper than that from observation. This may imply that other mechanisms may contribute to produce the relation found in King et al. (2013).

\subsection{A Simple Analysis}

The results presented in Figure 2 can be well understood on the basis of the light curves presented in Figure 1, especially those in the top panel. Taking the jet emission at frequency $10^{10} \mathrm{~Hz}$ as an example, it is shown that the luminosity of jet emission at this frequency keeps almost constant whereas the luminosity of disk emission exhibits strong variability. Therefore, the relation corresponding to this frequency runs parallel with the axis of disk emission. This behavior can be found in Figure 2 for the data with the symbol " $\triangle$ " . The reason for the correlation running as the symbol " $\star$ " is as follows. According to the models presented in Section 2, the variability of disk emission and jet power mainly depend on the fluctuation of accretion rate $\dot{M}$ and $\epsilon(r)$, i.e., Equations (2) and (7). These two equations reveal that the variabilities of disk emission and jet power are almost the same except for the amplitude of variability. Furthermore, the variability of jet emission is modulated by the fluctuation of jet power. Then, the variability of jet emission should be more or less in a way that works as the variability of jet power and thus disk emission. That is to say, a correlation 
between disk emission and jet emission should be naturally expected. If the variability of jet emission well tracks the variability of jet power, the variability of jet emission would follow that of disk emission except for the amplitude of variability. This behavior can be found in the top panel of Figure 1 with cyan color light curve $\left(\nu=10^{13} \mathrm{~Hz}\right)$ or in the bottom right panel of Figure 2 ,

As discussed above, a correlation of disk emission and jet emission is naturally expected owing to the fact that the jet power modulates the jet emission. However, why the variability of jet emission at different frequency is different? Since the jet emission at different frequency shares the same jet power, the different behavior of variability should be only associated with the emissivity profile of these emission. Figure 5 shows the emissivity profile of jet emission for different observed frequency in the accretion system with $M_{\mathrm{BH}}=10 M_{\odot}$, i.e., $10^{10} \mathrm{~Hz}$ (solid curve), $10^{11} \mathrm{~Hz}$ (dashed curve), $10^{12} \mathrm{~Hz}$ (dotted curve), and $10^{13} \mathrm{~Hz}$ (dot-dashed curve), respectively. In this figure, $I_{\nu}(z, t)$ is the jet emissivity at frequency $\nu$ from location $z$ observed at time $t$. Based on this figure, the luminosity of jet emission can be described as

$$
\begin{aligned}
L_{\mathrm{jet}}(\nu, t) & \propto \int_{z_{0}}^{z_{\mathrm{out}}} I_{\nu}\left(z, t-\left(z_{\mathrm{out}}-z\right) \cos \psi / c\right) z \phi d z \\
& \sim \int_{Z_{\mathrm{p}}-\delta Z_{p} / 2}^{Z_{\mathrm{p}}+\delta Z_{p} / 2} I_{\nu}\left(z, t-\left(z_{\mathrm{out}}-z\right) \cos \psi / c\right) z \phi d z
\end{aligned}
$$

where $z_{\text {out }}$ is the outer location of jet emission, $Z_{\mathrm{p}}(\nu)$ is the location of peak emissivity, $\delta Z_{p} \sim W_{\log } Z_{\mathrm{p}}$, and $W_{\log }$ is the logarithmic width of emissivity profile. It can be expected that the variability of $L_{\text {jet }}(\nu, t)$ in the timescale $\delta t \lesssim \delta Z_{p} / v_{\text {jet }}$ would be suppressed according to Equation (8). This behavior has been reported in the observation of GX $339-4$ (see Figure 3 of Casella et al. 2010 and Equation (15) of Lin et al. 2012). As shown in Figure 5 , the larger value of $Z_{\mathrm{p}}$ corresponds to the lower value of observed frequency. In addition, the value of $W_{\log }$ is almost the same for different observed frequency. Then, the lower value of $\nu$ would cause a stronger suppression of the variability. This can be found in Figure 1, where the variability of jet emission at low frequency (such as $10^{10} \mathrm{~Hz}$ ) is obviously weaker than those at high frequency (such as $10^{13} \mathrm{~Hz}$ ). This is the reason for the different variability behavior of jet emission observed at different $\nu$.

Equation (8) reveals that the variability of jet emission in the long timescale $\delta t>$ $\delta Z_{p} / v_{\text {jet }}$ would follow that of $I_{\nu}$ and thus $P_{\text {jet }}$ except for the amplitude of variability. In the work of Heinz \& Sunyaev (2003), the relation of $L_{\text {jet }}(\nu) \propto \dot{M}^{17 / 12}$ was derived for typical flat-spectrum core-dominated radio jets. Our simulations with a steady jet power confirm this relation but with a slightly different index for different $\nu$. Then, one can expect that the relation of disk emission and jet emission in the long timescale $\delta t>\delta Z_{p} / v_{\text {jet }}$ would be $L_{\text {jet }}(\nu) \propto L_{\mathrm{X}}^{17 / 24}$, where $L_{\mathrm{X}} \propto \dot{M}^{2}$ is adopted. This behavior can be found in the top 
left panel of Figure 4. For the jet emission at $\nu=10^{10} \mathrm{~Hz}$ from the accretion system with $M_{\mathrm{BH}}=10 M_{\odot}, \delta Z_{p} / v_{\text {jet }} \sim 100 \mathrm{~s}$ can be estimated based on Figure 5 . Then, the variability of jet emission with timescale $\delta t<100$ s would be suppressed, and that with timescale $\delta t>100 \mathrm{~s}$ will follow the variability of $P_{\text {jet }}$. This is the reason for the different behaviors in the top left panel of Figure 2 and that of Figure 4. The increase of slope in Figure 3 can also be understood as follows. In Figure 3, $\Delta t \propto M_{\mathrm{BH}}$ is adopted. However, the increase rate of $Z_{P}\left(\nu=10^{10} \mathrm{~Hz}\right) / v_{\text {jet }}$ with $M_{\mathrm{BH}}$ does not follow that of $\Delta t$. Then, the variability of jet emission in the accretion system with larger $M_{\mathrm{BH}}(\Delta t)$ would present a better tracing behavior for the jet power compared to the case with lower $M_{\mathrm{BH}}(\Delta t)$.

As discussed in Sections 2.1 and 2.2, the value of $\gamma($ or $\mu+s)$ may affect the variability

of disk emission (jet power) in the timescale $\delta t \lesssim t_{\text {vis,in }}$ (see Figure 2 of Arévalo \& Uttley 2006), where $t_{\mathrm{vis}, \text { in }}$ is the viscous timescale of ADAF in the inner region. For the case in the present work, however, the timescale $\delta t \sim \delta Z_{p} / v_{\text {jet }}$ (see Figure 5) is significantly larger than $t_{\mathrm{vis}, \mathrm{in}}$. Then, the value of $\gamma($ or $\mu+s)$ may not have significant effect on the relations presented in Figures 24,

\section{Conclusions and Discussion}

The variability of accretion rate, which is believed to induce the significant aperiodic variability of X-ray emission from disk, may affect the energy injection of jet from accreting process. Owing to this behavior, correlations between X-ray emission from disk and jet emission may be formed even if the mean value of X-ray luminosity keeps constant. These correlations are found in this work. It is shown that the correlation may be different from that of the fundamental plane. The fitted results show that the slope of correlation may increase with increasing observed frequency of jet emission. Taking an accretion system with $M_{\mathrm{BH}}=10 M_{\odot}$ as an example, it is shown that the slope of correlation varies from 0 to $\sim 0.58$ for the jet observed frequency varying from $10^{10} \mathrm{~Hz}$ to $10^{13} \mathrm{~Hz}$, where $L_{\mathrm{X}} \propto \dot{M}^{2}$ and total duration of observation is 100s. For the luminosities spacing with 3 days, which are used to estimate the fundamental plane in a practical context, the slope of relation decreases with increasing black hole mass. The shallow behaviour of our found relations is due to the suppression of variability in the jet emission compared with that in the disk emission. These results suggest that luminosities used to estimate the fundamental plane should cover an appropriate timescale, in which the variability of jet emission is not significantly suppressed compared with that of disk emission.

The shallow relations found in this work may appear in the observations. We compare the relations found in this paper with that of observations (King et al. 2013). In the 
work of King et al. (2013), a shallow relation, i.e., $\log \left(L_{\text {jet }} / \overline{L_{\text {jet }}}\right) \propto 0.06 \log \left(L_{\mathrm{X}} / \overline{L_{\mathrm{X}}}\right)$, has been reported in the observation of NGC 4395. In our work, we also find a relation, i.e., $\log \left(L_{\text {jet }} / \overline{L_{\text {jet }}}\right) \propto 0.19 \log \left(L_{\mathrm{X}} / \overline{L_{\mathrm{X}}}\right)$, which is shallower than that of the fundamental plane but still steeper than that of King et al. (2013). The deviation of our result with that of observation may imply that other mechanisms may contribute to produce the observed shallower relation. Besides the low value of relation slope found in observations (Bell et al. 2011; King et al. 2013), several sources, such as NGC 4051 (King et al. 2011), 3C 120 (Chatteriee et al. 2009), and 3C 111 (Chatterjee et al. 2011), show negative relation between $L_{\mathrm{X}}$ and $L_{\mathrm{R}}$. In these sources, the accretion rate is close to or larger than $0.01 \dot{M}_{\text {Edd }}$, around which the transition from low/hard state to high/soft state appears in BHBs (e.g., Yu \& Yan 2009; Dunn et al. 2010). Then, the negative relations may be due to the suppression of jet production in the high/soft state (Maccarone et al. 2003; Sikora et al. 2007; Coriat et al. 2011; Russell et al. 2011). This mechanism may work in NGC 4395, of which the accretion rate is $\sim 0.01 \dot{M}_{\text {Edd }}$. The radiative efficiency may increase in the state transition from low/hard state to high/soft state (e.g., Xie \& Yuan 2012). In this case, relation of $L_{\mathrm{X}}$ and $L_{\text {jet }}$ may be steeper than that of the fundamental plane. This behaviour has been found in some BHBs and AGNs (e.g., Coriat et al. 2011; Corbel et al. 2013; Dong et al. 2014; Panessa et al. 2015). However, this mechanism could not explain the shallower relation found in King et al. (2013) since it steepens the relation. It should be noted that the models presented in Section 2 may be better applied to a source in the low/hard state or high/soft state rather than in a transition state, where the suppression of jet and the increase of radiative efficiency may appear.

We thank the anonymous referee for beneficial suggestions to improve the paper. This work is supported by the National Basic Research Program of China (973 Program, grant No. 2014CB845800), the National Natural Science Foundation of China (Grant No. 11403005, 11025313, 11222328, 11333004), the Guangxi Science Foundation (Grant No. 2014GXNSFBA118004, 2013GXNSFFA019001), the Project Sponsored by the Scientific Research Foundation of Guangxi University (Grant No. XJZ140331), and the Key Scientific Research Project in Universities of Henan Province (Grant No. 15A160001).

\section{REFERENCES}

Abramowicz, M. A., Czerny, B., Lasota, J. P., \& Szuszkiewicz, E. 1988, ApJ, 332, 646

Arévalo, P., \& Uttley, P. 2006, MNRAS, 367, 801

Armitage, P. J., \& Natarajan, P. 1999, ApJ, 523, L7 
Balbus, S. A., \& Hawley, J. F. 1991, ApJ, 376, 214

Balbus, S. A., \& Hawley, J. F. 1998, RvMP, 70, 1

Begelman, M. C., Blandford, R. D., \& Rees, M. J. 1984, RvMP, 56, 255

Bell, M. E., Tzioumis, T., Uttley, P., et al. 2011, MNRAS, 411, 402

Blandford, R. D., \& Payne, D. G. 1982, MNRAS, 199, 883

Blandford, R. D., \& Znajek, R. L. 1977, MNRAS, 179, 433

Cao, X. 2002, MNRAS, 332, 999

Casella, P., Maccarone, T. J., O’Brien, K., et al. 2010, MNRAS, 404, L21

Chandrasekhar, S. 1960, PNAS, 46, 253

Chatterjee, R., Marscher, A. P., Jorstad, S. G., et al. 2009, ApJ, 704, 1689

Chatterjee, R., Marscher, A. P., Jorstad, S. G., et al. 2011, ApJ, 734, 43

Corbel, S., Nowak, M. A., Fender, R. P., Tzioumis, A. K., \& Markoff, S. 2003, A\&A, 400, 1007

Corbel, S., Coriat, M., Brocksopp, C., et al. 2013, MNRAS, 428, 2500

Coriat, M., Corbel, S., Prat, L., et al. 2011, MNRAS, 414, 677

Dong, A.-J., Wu, Q., \& Cao, X.-F. 2014, ApJ, 787, LL20

Dunn, R. J. H., Fender, R. P., Körding, E. G., Belloni, T., \& Cabanac, C. 2010, MNRAS, 403, 61

Falcke, H., Körding, E., \& Markoff, S. 2004, A\&A, 414, 895

Gallo, E., Fender, R. P., \& Pooley, G. G. 2003, MNRAS, 344, 60

Ghosh, P., \& Abramowicz, M. A. 1997, MNRAS, 292, 887

Guan, X., \& Gammie, C. F. 2009, ApJ, 697, 1901

Haardt, F., \& Maraschi, L. 1993, ApJ, 413, 507

Heil, L. M., Vaughan, S., \& Uttley, P. 2012, MNRAS, 422, 2620

Heinz, S., \& Sunyaev, R. A. 2003, MNRAS, 343, L59 
Heyvaerts, J., Priest, E. R., \& Bardou, A. 1996, ApJ, 473, 403

Janiuk, A., \& Czerny, B. 2007, A\&A, 466, 793

King, A. L., Miller, J. M., Cackett, E. M., et al. 2011, ApJ, 729, 19

King, A. L., Miller, J. M., Reynolds, M. T., et al. 2013, ApJ, 774, L25

King, A. R., Pringle, J. E., West, R. G., \& Livio, M. 2004, MNRAS, 348, 111

Kotov, O., Churazov, E., \& Gilfanov, M. 2001, MNRAS, 327, 799

Li, S.-L., \& Cao, X. 2012, ApJ, 753, 24

Liang, E. P. T., \& Price, R. H. 1977, ApJ, 218, 247

Lin, D.-B., Gu, W.-M., Liu, T., \& Lu, J.-F. 2012, MNRAS, 421, 308

Lin, D.-B., Gu, W.-M., Liu, T., Sun, M.-Y., \& Lu, J.-F. 2012, ApJ, 761, 29

Livio, M., Ogilvie, G. I., \& Pringle, J. E. 1999, ApJ, 512, 100

Lubow, S. H., Papaloizou, J. C. B., \& Pringle, J. E. 1994, MNRAS, 267, 235

Lyubarskii, Y. E. 1997, MNRAS, 292, 679

Nemmen, R. S., Bower, R. G., Babul, A., \& Storchi-Bergmann, T. 2007, MNRAS, 377, 1652

Maccarone, T. J., Gallo, E., \& Fender, R. 2003, MNRAS, 345, L19

Markoff, S., Falcke, H., \& Fender, R. 2001, A\&A, 372, L25

Martínez-Sansigre, A., \& Rawlings, S. 2011, MNRAS, 414, 1937

Mayer, M., \& Pringle, J. E. 2006, MNRAS, 368, 379

Merloni, A., Heinz, S., \& Di Matteo, T. 2003, MNRAS, 345, 1057

Merloni, A., Körding, E., Heinz, S., et al. 2006, New A, 11, 567

Meier, D. L. 2001, ApJ, 548, L9

Moderski, R., \& Sikora, M. 1996, MNRAS, 283, 854

Narayan, R., \& Yi, I. 1994, ApJ, 428, L13

Nemmen, R., Storchi-Bergmann, T., \& Eracleous, M. 2011, arXiv:1112.4640 
Panessa, F., Tarchi, A., Castangia, P., et al. 2015, MNRAS, 447, 1289

Piran, T. 1999, Phys. Rep., 314, 575

Russell, D. M., Miller-Jones, J. C. A., Maccarone, T. J., et al. 2011, ApJ, 739, LL19

Shakura, N. I., \& Sunyaev, R. A. 1973, A\&A, 24, 337

Sikora, M., Stawarz, L., \& Lasota, J.-P. 2007, ApJ, 658, 815

Spada, M., Ghisellini, G., Lazzati, D., \& Celotti, A. 2001, MNRAS, 325, 1559

Sun, M.-Y., Jin, Y.-L., Gu, W.-M., et al. 2013, ApJ, 776, 118

Uttley, P., \& McHardy, I. M. 2001, MNRAS, 323, L26

Uttley, P., McHardy, I. M., \& Vaughan, S. 2005, MNRAS, 359, 345

Velikhov, E. P. 1959, Sov. Phys. JETP, 36, 995

Wu, Q., Yuan, F., \& Cao, X. 2007, ApJ, 669, 96

Wu, Q., \& Cao, X. 2008, ApJ, 687, 156

Xie, F.-G., \& Yuan, F. 2012, MNRAS, 427, 1580

Yu, W., \& Yan, Z. 2009, ApJ, 701, 1940

Yuan, F., Cui, W., \& Narayan, R. 2005, ApJ, 620, 905

Yuan, F., \& Narayan, R. 2014, ARA\&A, 52, 529 


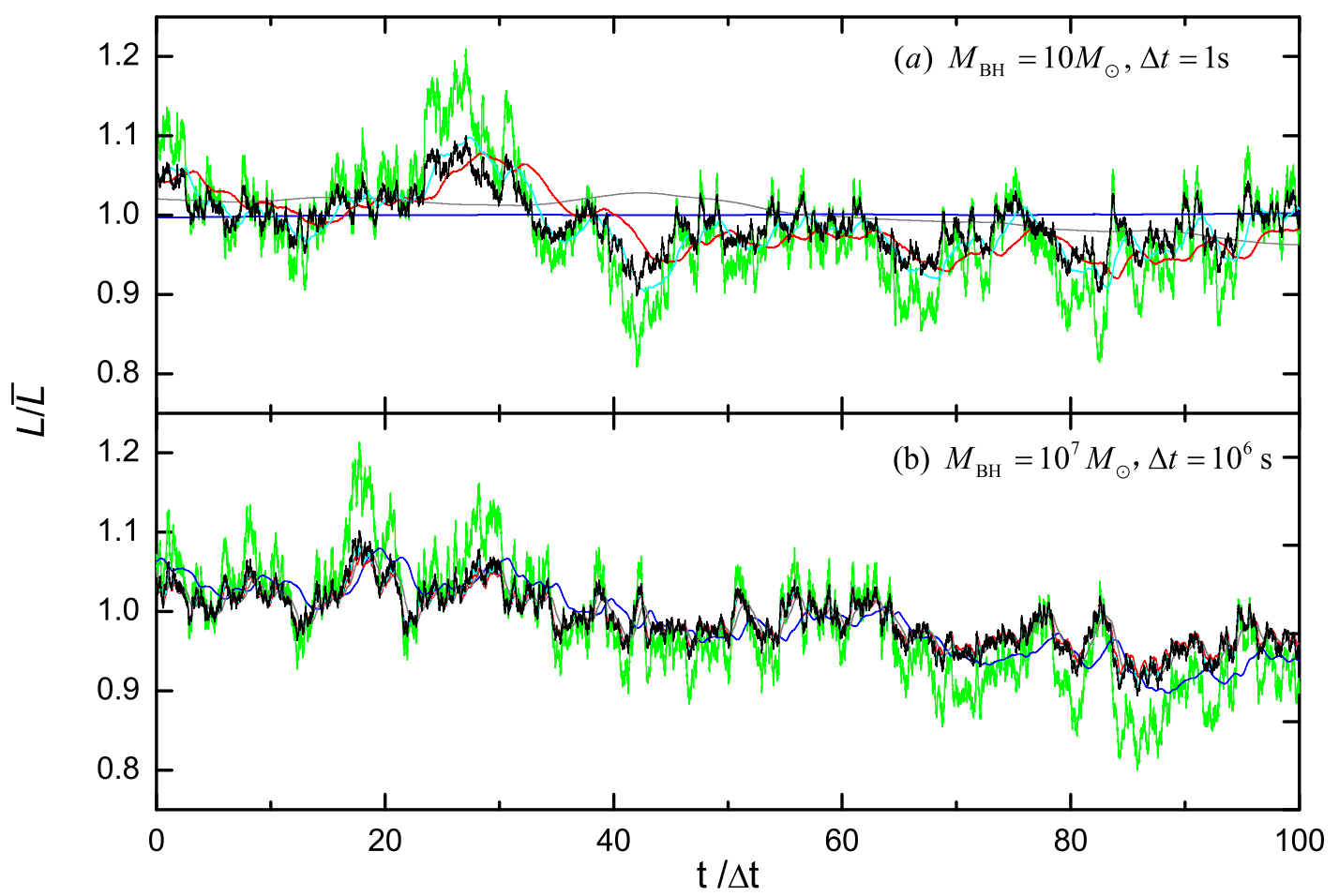

Fig. 1. - Light curves of disk emission and jet emission with different black hole mass. The top panel is for $M_{\mathrm{BH}}=10 M_{\odot}$ and the bottom panel is for $M_{\mathrm{BH}}=10^{7} M_{\odot}$. The blue, gray, red, and cyan curves represent the light curves of jet emission at frequency $10^{10} \mathrm{~Hz}, 10^{11} \mathrm{~Hz}$, $10^{12} \mathrm{~Hz}$, and $10^{13} \mathrm{~Hz}$, respectively. The light curves of disk emission and $P_{\text {jet }}$ are presented with green and black curves, respectively. 


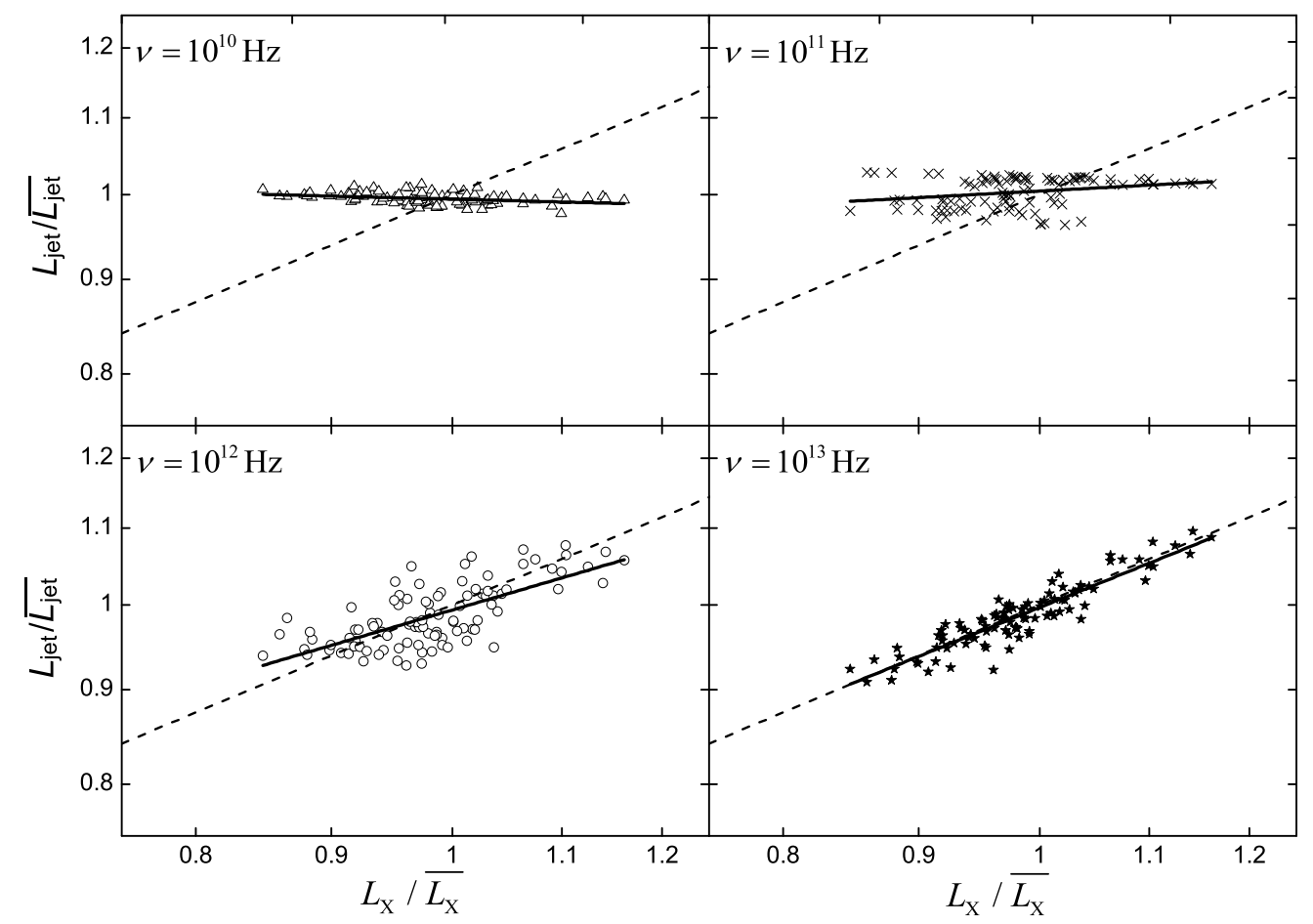

Fig. 2.- Relations between disk emission and jet emission, where $\triangle, \times, \circ$, and $\star$ represent the relation with jet emission at frequency $10^{10} \mathrm{~Hz}, 10^{11} \mathrm{~Hz}, 10^{12} \mathrm{~Hz}$, and $10^{13} \mathrm{~Hz}$, respectively. The solid line and dashed line in each panel represent the logarithmic linear fitting result and the relation of the fundamental plane with a given black hole mass, respectively. 


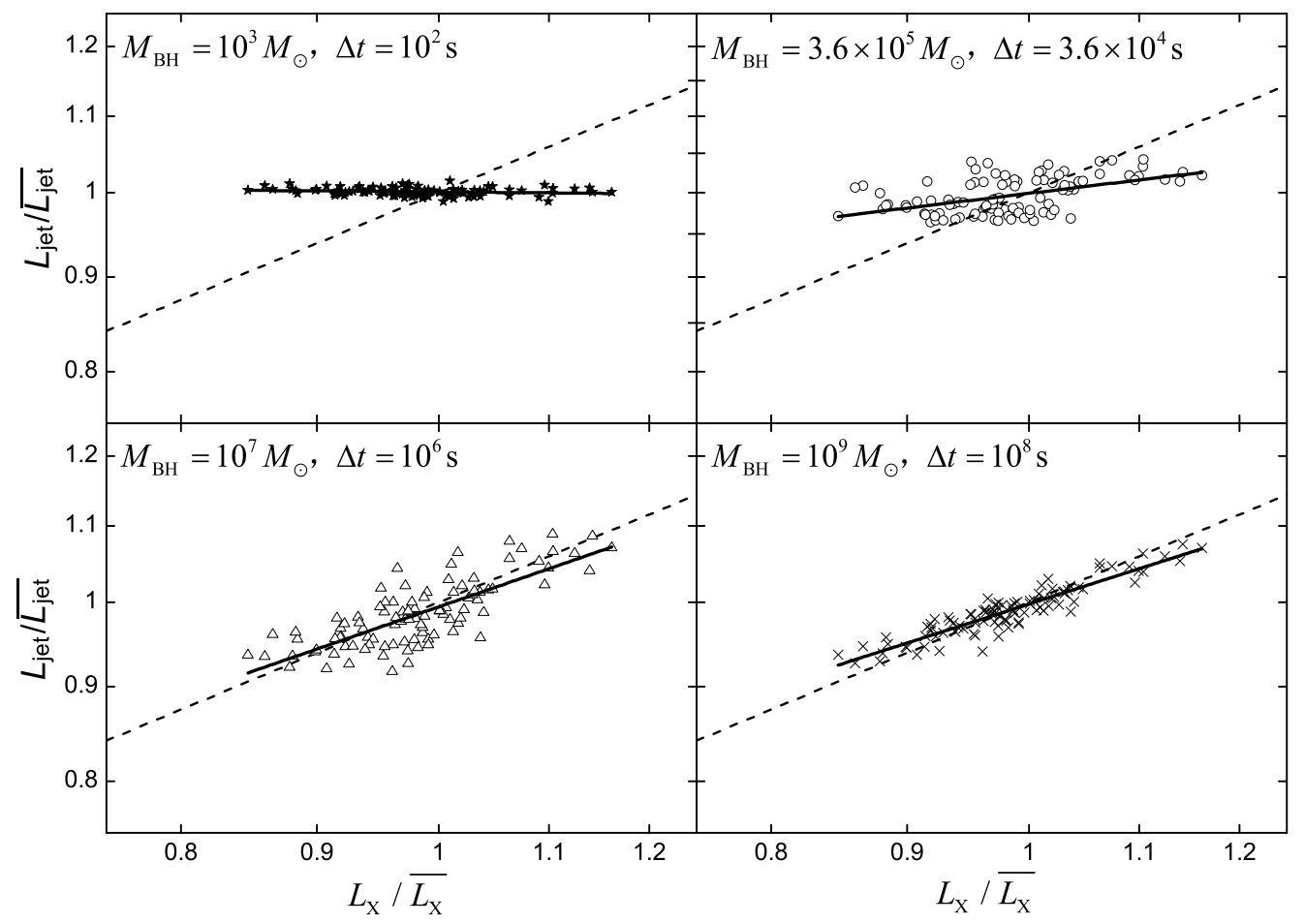

Fig. 3.- Relations between disk emission and jet emission at frequency $10^{10} \mathrm{~Hz}$, where " $\star$ ", "०", " $\triangle$ ", and " $\times$ " are the data from an accretion system with $M_{\mathrm{BH}}=10^{3} M_{\odot}\left(\Delta t=10^{2} \mathrm{~s}\right)$, $3.6 \times 10^{5} M_{\odot}\left(\Delta t=3.6 \times 10^{4} \mathrm{~s}\right), 10^{7} M_{\odot}\left(\Delta t=10^{6} \mathrm{~s}\right)$, and $10^{9} M_{\odot}\left(\Delta t=10^{8} \mathrm{~s}\right)$, respectively The solid line and dashed line in each panel represent the logarithmic linear fitting result and the relation of the fundamental plane with a given black hole mass, respectively. 


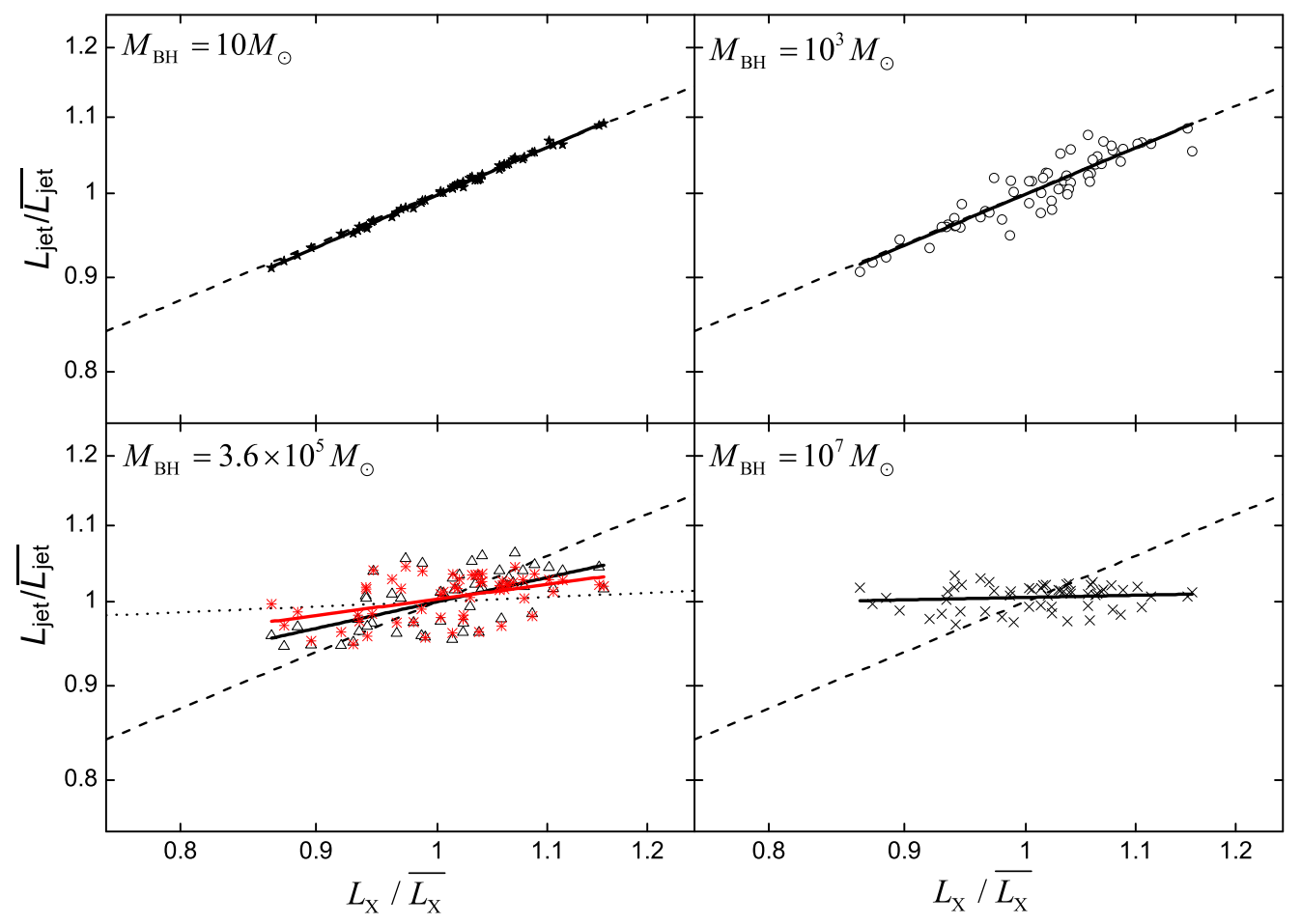

Fig. 4.- Relations between disk emission and jet emission at frequency $10^{10} \mathrm{~Hz}$, where " $\star$ ", "०", " $\triangle$ ", and " $\times$ " are the data from an accretion system with $M_{\mathrm{BH}}=10 M_{\odot}, 10^{3} M_{\odot}$, $3.6 \times 10^{5} M_{\odot}$, and $10^{7} M_{\odot}$, respectively. The data are the averaged lumonisities in $\Delta t=1 \mathrm{ks}$ with a spacing time of 3 days. The solid line and dashed line in each panel represent the logarithmic linear fitting result and the relation of the fundamental plane with a given black hole mass, respectively. The red solid line represents the logarithmic linear fitting result about the data with $L_{\text {jet }}$ observed at $\nu=5 \mathrm{GHz}$, which is shown with red "**. The dotted line in the bottom left panel represents the relation of $\log \left(L_{\text {jet }} / \overline{L_{\text {jet }}}\right) \propto 0.06 \log \left(L_{\mathrm{X}} / \overline{L_{\mathrm{X}}}\right)$, which was found in NGC 4395 (King et al. 2013). 


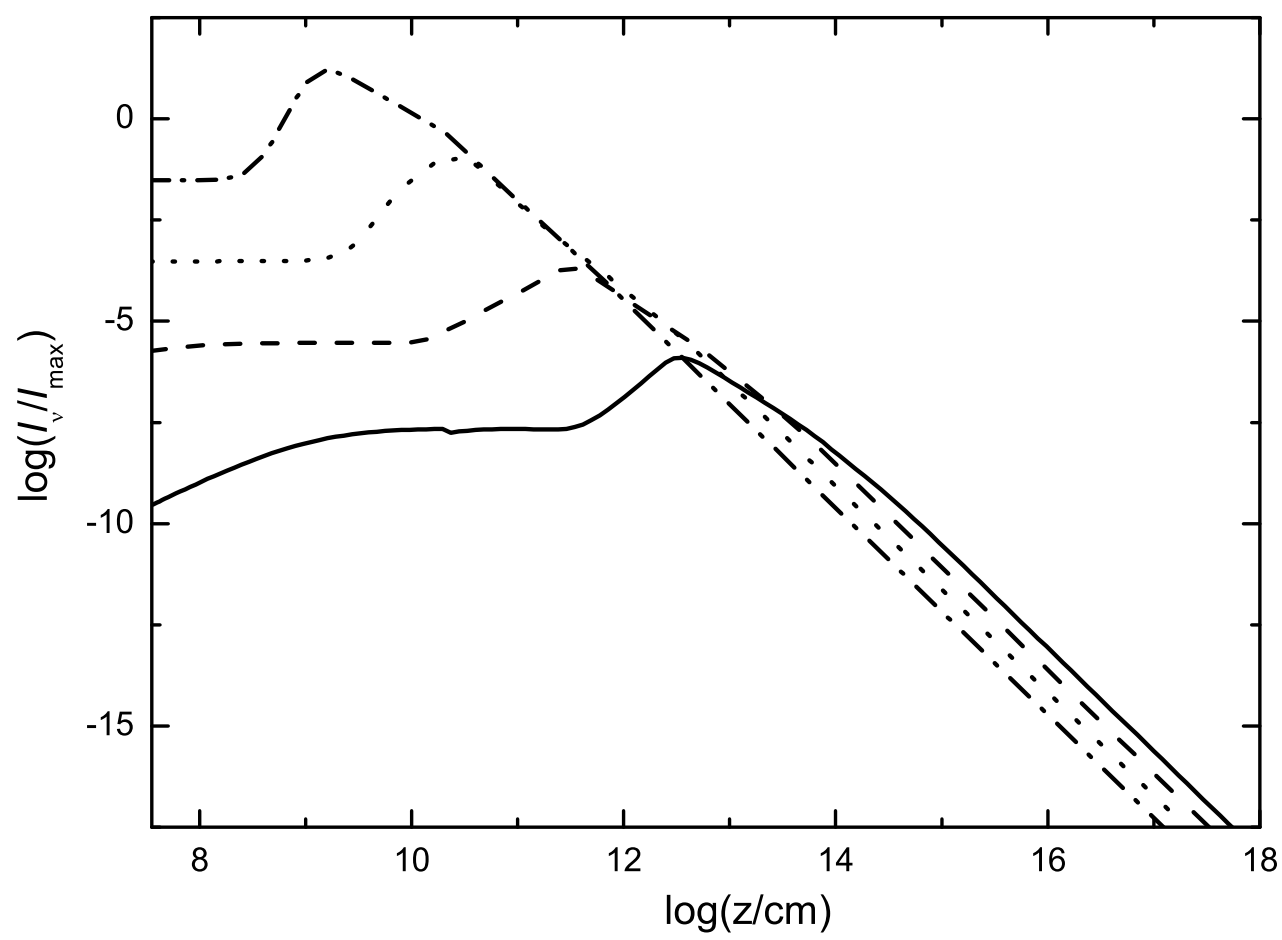

Fig. 5.- Emissivity profile of jet emission at frequency $10^{10} \mathrm{~Hz}$ (solid curve), $10^{11} \mathrm{~Hz}$ (dashed curve), $10^{12} \mathrm{~Hz}$ (dotted curve), and $10^{13} \mathrm{~Hz}$ (dot-dashed curve), respectively. 